\title{
Ursolic acid activates the apoptosis of prostate cancer via ROCK/PTEN mediated mitochondrial translocation of cofilin-1
}

\author{
DAWEI MU, GAOBIAO ZHOU, JIANYE LI, BIN SU and HEQING GUO \\ Department of Urology, The General Hospital of The Air Force of The Chinese People's Liberation Army, \\ Beijing 100142, P.R. China
}

Received September 3, 2015; Accepted October 20, 2017

DOI: $10.3892 / 01.2017 .7689$

\begin{abstract}
Ursolic acid has various pharmacological activities, and can reduce blood fat as well as having antihepatic, antitumoral, anti-inflammatory and antiviral properties. However, the pro-apoptotic mechanism by which ursolic acid influences human prostate cancer requires additional study. The aim of the present study was to assess whether ursolic acid activates the apoptosis of prostate cancer and to investigate the mechanism by which the Rho-associated protein kinase 1 (ROCK1)/phosphatase and tensin homolog (PTEN) signaling pathway performs a role in ursolic acid-mediated cofilin-1 to induce apoptosis in human prostate cancer. Firstly, the present study determined the pro-apoptotic mechanism by which ursolic acid influences the cell proliferation and apoptosis of human prostate LNCaP cancer cells. Caspase-3/9 activities and ROCK1, PTEN, Cofilin-1 and cytochrome c protein expression levels were also analyzed. In the present study, it is reported that the pro-apoptotic mechanism of ursolic acid potently suppressed the cell proliferation of human prostate $\mathrm{LNCaP}$ cancer cells. The present study revealed that the mediation of ROCK1/PTEN-cofilin-1/cytochrome c protein expression activates caspase-3/9 activities which subsequently induced the apoptosis of human prostate cancer cells. In conclusion, these findings demonstrated that ursolic acid activates the apoptosis of prostate cancer via ROCK/PTEN mediated cofilin-1/cytochrome $\mathrm{c}$ which mediated caspase-3/9 activities.
\end{abstract}

\section{Introduction}

In western countries, prostate cancer is the most common type of non-malignant skin tumor and is the leading cause

Correspondence to: Mr. Heqing Guo, Department of Urology, The General Hospital of The Air Force of The Chinese People's Liberation Army, 30 Fucheng Road, Haidian, Beijing 100142, P.R. China

E-mail: ping493822407@126.com

Key words: ursolic acid, prostate cancer, Rho-associated protein kinase 1/phosphatase and tensin homolog, cofilin-1 of cancer-associated mortality (1). Castration remains the standard method for the treatment of prostate cancer, particularly metastatic prostate cancer, however it cannot successfully treat this disease (2). It is predicted that $\sim 80 \%$ of patients with prostate cancer experience a recurrence of clinical symptoms or a change to the volume of prostate cancer following the removal of the androgen (3). However, following a median treatment period of 18-24 months, almost all patients enter the hormone independent phase of prostate cancer (4).

Cofilin is a type of actin-binding protein that exists in eukaryotes, and has a low molecular weight (5). The Cofilin-1 gene is located on hormone 11q13 and is expressed in various non-muscular tissues, particularly in the liver and brain (6). The progression of tumor cells occurs in a complexed micro-environment through migration (7), by invasion into pseudopods. During the invasion and metastasis of tumor cells, Cofilin-1 performs an essential role in the remodeling of the actin skeleton (8). A previous study suggested that expression levels of Cofilin-1 and changes to cellular activities have been identified in tissues of oral squamous cell carcinoma, renal cell carcinoma and ovarian cancer as well as in in vitro cultured carcinoma cell lines (9).

Ursolic acid (UA) is a pentacyclic triterpenoid, with the chemical name, molecular formula and molecular weight of (3ß)-3-Hydroxy-urs-12-en-28-oic acid, $\mathrm{C}_{30} \mathrm{H}_{48} \mathrm{O}_{3}$ and 456, respectively (10). UA has a wide distribution, and exists in the form of dissociation or glycoside in Sambucus chinensis, Folium eriobotryae, bearberry, glossy privet fruits, plantain herbs, hawthorn, selfheal and Oldenlandia diffusa (11). UA has low toxicity and few side effects, and has various pharmacological activities including anti-hepatitic, anti-tumor, anti-inflammatory, anti-viral and reducing blood lipids (12-14). Additional investigation into the effect of UA on the apoptosis of prostate cancer and its possible signal transduction pathway may provide a potential drug target for the clinical treatment of patients with prostate cancer.

\section{Materials and methods}

Cell culture. Human prostate cancer LNCaP cells were cultured in RPMI-1640 medium (Invitrogen; Thermo Fisher Scientific, Inc., Waltham, MA, USA) supplemented with $10 \%$ fetal bovine serum (Invitrogen; Thermo Fisher Scientific, Inc.), 
$100 \mathrm{~g} / \mathrm{ml}$ streptomycin and $100 \mathrm{U} / \mathrm{ml}$ penicillin and maintained at a high humidity, at $37^{\circ} \mathrm{C}$ under $5 \% \mathrm{CO}_{2}$.

Cell viability analysis. Cell viability was measured using an MTT assay (Sigma-Aldrich; Merck KGaA, Darmstadt, Germany). LNCaP cells were seeded in 96-well plates at $1 \times 10^{4}$ cells/well and incubated with varying concentrations of ursolic acid (0-80 $\mu \mathrm{M}$, purity $\geq 98.5 \%$; Sigma-Aldrich; Merck $\mathrm{KGaA}$ ), and incubated for 24,48 or $72 \mathrm{~h}$ at $37^{\circ} \mathrm{C}$. Cells incubated with $0 \mu \mathrm{M}$ ursolic acid were used as controls. Following the incubation, $50 \mu \mathrm{l}$ of the MTT assay was added into each well and the cells were incubated for an additional $4 \mathrm{~h}$ at $37^{\circ} \mathrm{C}$. Following a 10-min wash with DMSO, the absorbance was detected at a wavelength of $490 \mathrm{~nm}$ using a microplate reader (Bio-Rad Laboratories, Inc., Hercules, CA, USA).

Western blot analysis. LNCaP cells were seeded in 6-well plates at a density of $1 \times 10^{6}$ cells/well $(n=3)$, incubated with varying concentrations of ursolic acid (0-80 $\mu \mathrm{M})$ and incubated for $48 \mathrm{~h}$ at $37^{\circ} \mathrm{C}$. The LNCaP cells were prepared using a Proteo JET cytoplasmic protein extraction kit (Fermentas; Thermo Fisher Scientific, Inc.). Protein concentration was measured using bicinchoninic acid (BCA; Beyotime Institute of Biotechnology, Haimen, China). Proteins $(50 \mu \mathrm{g}$ per lane) were loaded onto 10-12\% SDS-PAGE for separation by electrophoresis, transferred onto polyvinylidene difluoride (PVDF) membranes, and blocked using TBST (and $0.1 \%$ Tween-20) containing 5\% non-fat milk. The PVDF membranes were then incubated overnight at $4{ }^{\circ} \mathrm{C}$ with the following primary antibodies: Anti-ROCK (dilution, 1:1,000; cat. no. sc-33779); anti-phosphorylation-PTEN (dilution, 1:1,000; cat. no. sc-101789); anti-Cofilin-1 (dilution, 1:1,000; cat. no. sc-33779); anti-Cytochrome c (dilution, 1:3,000; cat. no. sc-7159); and $\beta$-actin (dilution, 1:1,000; cat. no. sc-7210) (all from Santa Cruz Biotechnology, Inc. Dallas, TX, USA). PVDF membranes were subsequently incubated with secondary antibody (1:2,000; cat. no. sc-2004; Santa Cruz Biotechnology, Inc. Dallas, TX, USA) for $1 \mathrm{~h}$ at $37^{\circ} \mathrm{C}$ and were visualized using BeyoECL Plusenhanced chemiluminescence (P0018, Beyotime Institute of Biotechnology) and analyzed using Image_Lab_3.0 (Bio-Rad Laboratories, Inc.).

Flow cytometric analysis of apoptosis. An Annexin V-fluorescein isothiocyanate (FITC)/propidium iodide (PI) kit (BD Biosciences, Franklin Lakes, NJ, USA) was used to measure apoptosis. LNCaP cells were seeded in 6-well plates at a density of $1 \times 10^{6}$ cells/well, incubated with varying concentrations of ursolic acid $(0-80 \mu \mathrm{M})$ and incubated for 48 h. The cells were collected, washed twice with cold PBS (Shanghai Yantuo Biological Technology Co., Ltd., Shanghai, China), and resuspended in $400 \mu \mathrm{l}$ of binding buffer. A total of $5 \mu \mathrm{l}$ of Annexin V-FITC was added into each well and cells were incubated for $10 \mathrm{~min}$ at $4^{\circ} \mathrm{C}$ in the dark. A total of $10 \mu \mathrm{l}$ of PI was then added and cells were cultured for an additional 5 min in the dark. Apoptosis was analysis using FACSCalibur 7.6.1 flow cytometry (BD Biosciences).

Analysis of caspase-3 and caspase-9 protease activity. Caspase-3 and caspase-9 protease activity was measured using Caspase 3 Activity Assay Kit and Caspase 9 Activity Assay
Kit (Promega Corporation, Madison, WI, USA). LNCaP cells were seeded in 96-well plates at $1 \times 10^{4}$ cells/well and incubated with varying concentrations of UA $(0-80 \mu \mathrm{M})$ for $48 \mathrm{~h}$ at $37^{\circ} \mathrm{C}$. Subsequently, $100 \mu 1$ of Caspase-Glo 3 or Caspase-Glo 9 reagent was added to each well and incubated at room temperature for 2 h. Caspase- 3 and caspase- 9 protease activity was measured using a TD 20/20 luminometer (Promega Corporation).

Statistical analysis. Data are presented as the mean \pm standard deviation and were analyzed using the statistical software SPSS version 11.0 (SPSS, Inc., Chicago, IL, USA). Statistical analysis was performed using a one way analysis of variance. $\mathrm{P}<0.05$ was considered to indicate a statistically significant difference.

\section{Results}

Ursolic acid suppresses cell proliferation of prostate cancer. The chemical structure of ursolic acid is shown in Fig. 1. The present study evaluated whether UA suppresses the cell proliferation of LNCaP prostate cancer using an MTT assay. When compared with $0 \mu \mathrm{M}$, ursolic acid caused a decrease in cell proliferation of LNCaP cells in a time- and dose-dependent manner (Fig. 2). The decrease in cell proliferation following treatments with: $50 \mu \mathrm{M}$ of ursolic acid for 1 day; $30-50 \mu \mathrm{M}$ ursolic acid for 2 days; and 20-50 $\mu \mathrm{M}$ of ursolic acid for 3 days were statistically significant (Fig. 2).

Ursolic acid activates ROCK of prostate cancer. To additionally investigate the effect of UA on the ROCK pathway of prostate cancer, the present study examined the protein expression of ROCK and cleaved ROCK in LNCaP cells. In comparison with the control group ( $0 \mu \mathrm{M}$ ursolic acid), ROCK protein expression in LNCaP cells was significantly reduced and cleaved ROCK protein expression was significantly elevated in the 20 and $40 \mu \mathrm{M}$ ursolic acid treatment groups (Fig. 3).

Ursolic acid activates PTEN of prostate cancer. To additionally investigate whether ursolic acid affects the PTEN pathway of prostate cancer, the present study detected the protein expression of phosphorylated PTEN (p-PTEN) in all the experimental groups. In comparison with the control group ( $0 \mu \mathrm{M}$ ursolic acid), the p-PTEK protein expression of $\mathrm{LNCaP}$ cells was significantly promoted by 20 or $40 \mu \mathrm{M}$ of ursolic acid (Fig. 3).

Ursolic acid activates cofilin-1 of prostate cancer. To improve the understanding of how ursolic acid affects cofilin-1 of prostate cancer, the present study detected cofilin- 1 in LNCaP cells. Cofilin-1 protein expression in cytoplasmic LNCaP cells was observed to be significantly enhanced by treatment with 20 or $40 \mu \mathrm{M}$ ursolic acid, compared with the control group (Fig. 4).

Ursolic acid activates cytochrome c of prostate cancer. The present study also examined the expression of cytochrome $\mathrm{c}$ in LNCaP cells in order to understand how ursolic acid affects cytochrome $\mathrm{c}$ in prostate cancer. As demonstrated in Fig. 4, there was a significant increase in cytochrome $\mathrm{c}$ protein 


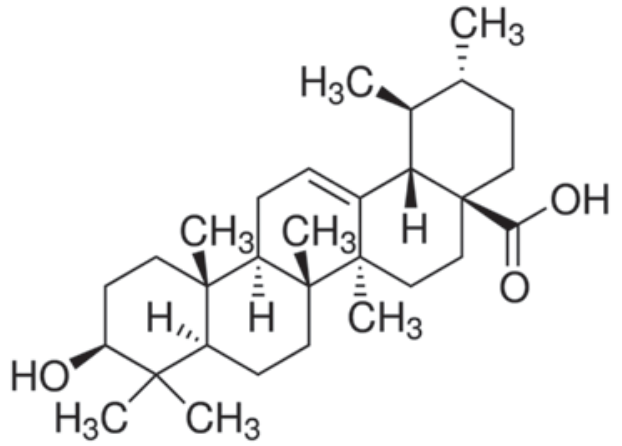

Figure 1. The chemical structure of ursolic acid.

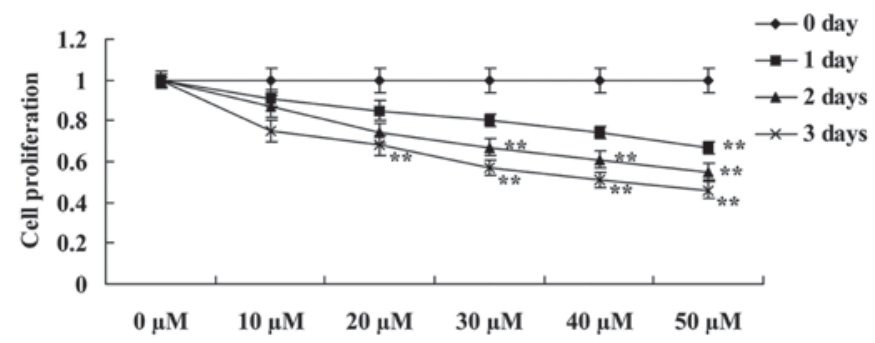

Figure 2. Ursolic acid suppresses the cell proliferation of prostate cancer. ${ }^{* *} \mathrm{P}<0.01$ compared with the $0 \mu \mathrm{M}$ ursolic acid (control) group.

A
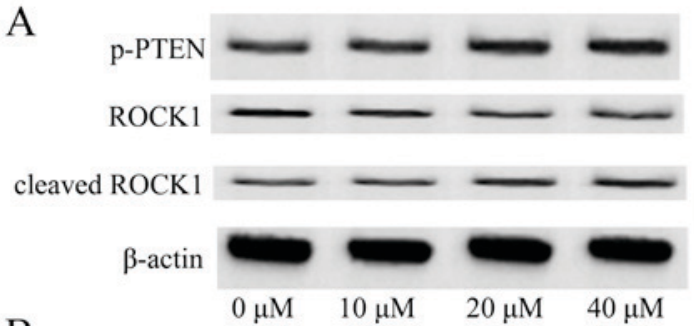

$\mathrm{B}$

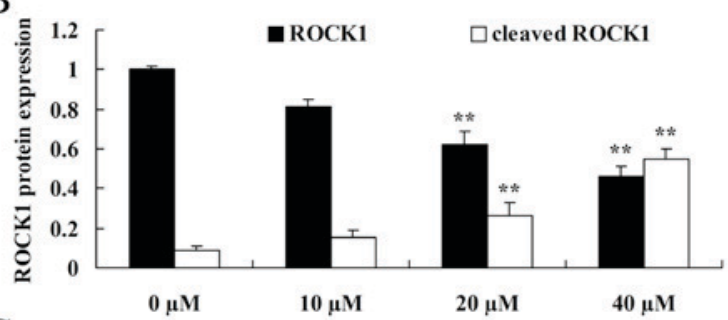

$\mathrm{C}$

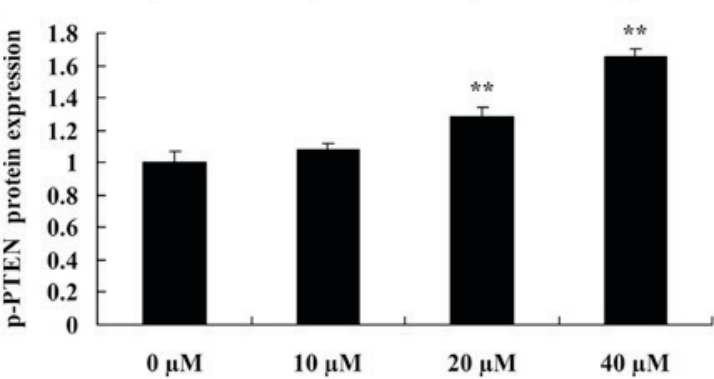

Figure 3. Ursolic acid activates cleaved ROCK and PTEN in prostate cancer. (A) Western blot analysis demonstrates that ursolic acid suppresses ROCK and activates cleaved ROCK protein expression. $\beta$-actin was used as the loading control. (B) Representation of the statistical analysis of ROCK and cleaved ROCK protein expression in prostate cancer LNCaP cells. (C) Statistical analysis of p-PTEK protein expression in prostate cancer LNCaP cells. ${ }^{* *} \mathrm{P}<0.01$ compared with the $0 \mu \mathrm{M}$ ursolic acid group. ROCK, Rho-associated protein kinase 1; PTEN, phosphatase and tensin homolog.

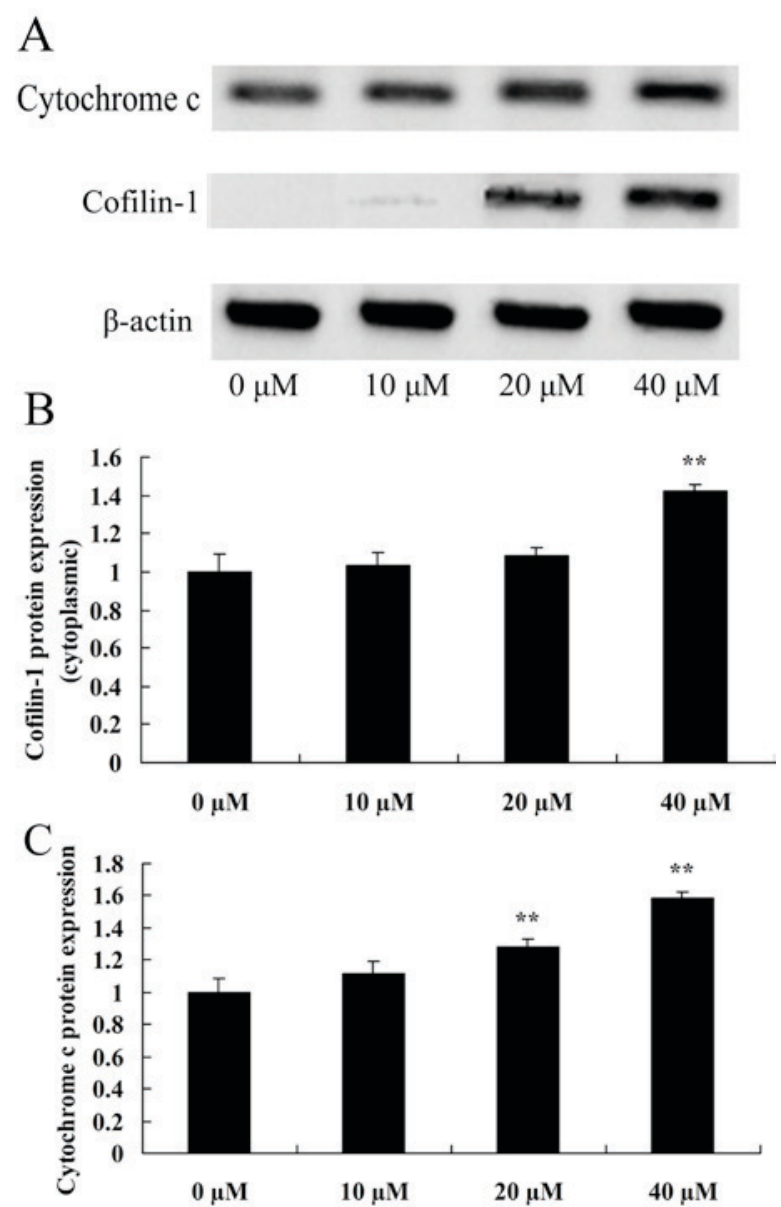

Figure 4. Ursolic acid activates Cofilin-1 and cytochrome c of prostate cancer of prostate cancer. (A) Western blot analysis demonstrating that high concentrations of ursolic acid activates cofilin-1 and cytochrome c protein expression. $\beta$-actin was used as the loading control. (B) Statistical analysis of cofilin-1 protein expression in cytoplasmic prostate cancer LNCaP cells. (C) Statistical analysis of Cytochrome c protein expression in prostate cancer LNCaP cells. ${ }^{* *} \mathrm{P}<0.01$ compared with $0 \mu \mathrm{M}$ ursolic acid group.

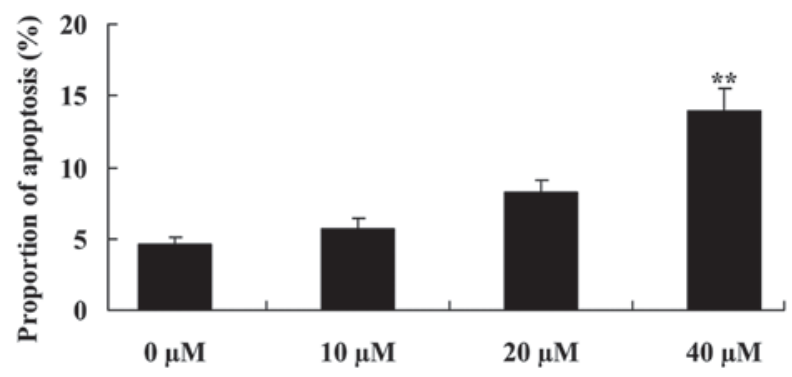

Figure 5. Results of flow cytometric analysis demonstrating how ursolic acid activates apoptosis of prostate cancer LNCaP cells. ${ }^{* *} \mathrm{P}<0.01$ compared with the $0 \mu \mathrm{M}$ ursolic acid group.

expression of LNCaP cells in the 20 and $40 \mu \mathrm{M}$ ursolic acid group in comparison with the control group.

Ursolic acid activates apoptosis of prostate cancer. The apoptosis of LNCaP cells was also investigated in order to investigate the effect of ursolic acid on prostate cancer cells. Fig. 5 demonstrates that treatments with 20 and $40 \mu \mathrm{M}$ 

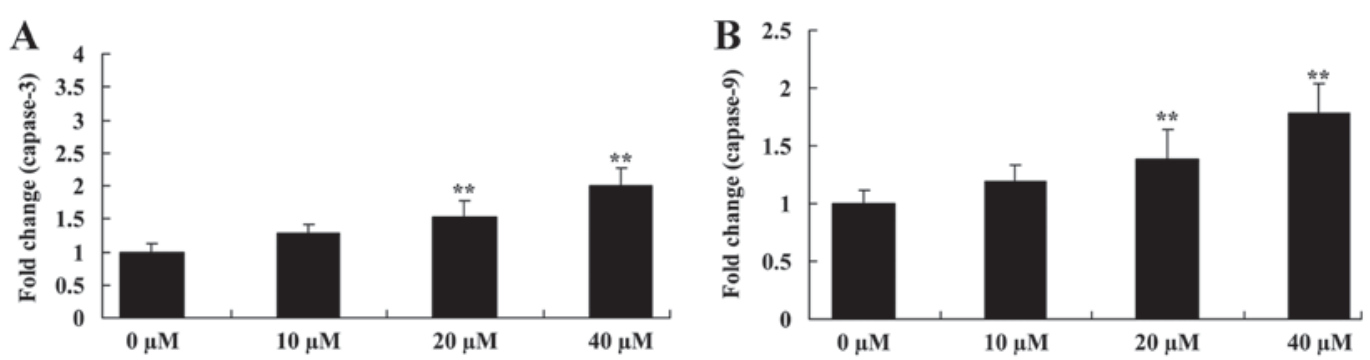

Figure 6. Ursolic acid activates the caspase-3 and caspase-9 activities of prostate cancer. Ursolic acid activates Caspase-3 and caspase-9 activities in prostate cancer LNCaP cells. " $\mathrm{P}<0.01$ compared with the $0 \mu \mathrm{M}$ ursolic acid group.

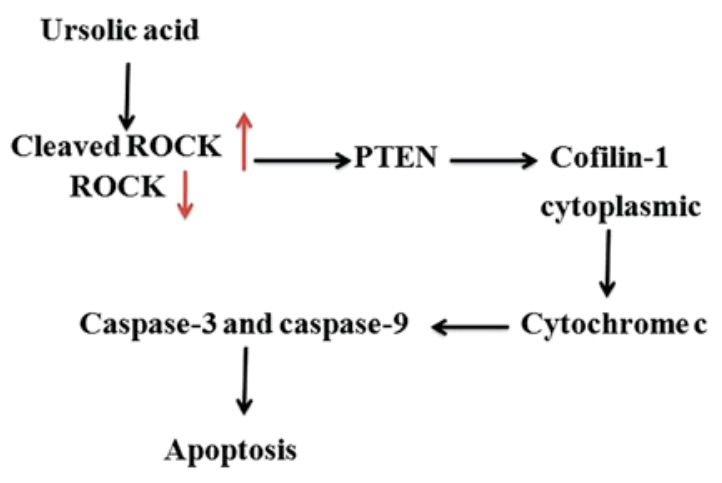

Figure 7. Mechanism by which ursolic acid activates the apoptosis of prostate cancer via ROCK/PTEN mediated mitochondrial translocation of cofilin-1. ROCK, rho-associated protein kinase 1; PTEN, phosphatase and tensin homolog.

ursolic acid significantly induced apoptosis of $\mathrm{LNCaP}$ cells in comparison with the $0 \mu \mathrm{M}$ ursolic acid group (control).

Ursolic acid activates caspase-3 and caspase-9 activities of prostate cancer. The present study also investigated the mechanism of apoptosis, by examining caspase- 3 and caspase- 9 activities of LNCaP cells. Compared with the $0 \mu \mathrm{M}$ ursolic acid group, caspase- 3 and caspase- 9 activities of $\mathrm{LNCaP}$ cells were significantly increased by the 20 and $40 \mu \mathrm{M}$ ursolic acid treatment groups (Fig. 6).

\section{Discussion}

In western countries, prostate cancer is the most common type of cancer for males and also the leading cause of cancer associated mortality (3). This is also now observed in China. Castration is an effective treatment option for early prostate cancer patients (15). In the present study, it was observed that ursolic acid suppressed cell proliferation and induced the apoptosis of LNCaP cells. Park et al (16) suggested that ursolic acid induced apoptosis in prostate cancer PC-3 cells via caspase-9 and -3. Zhang et al (17) suggested that ursolic acid inhibits the proliferation and promotes apoptosis in human ovarian cancer.

By inducing actin to connect with proteins and myosin, ROCK regulates protein phosphorylation through the contraction of actomyosin (18). The contraction of actomyosin is important for cell movement, and the inhibition of ROCK activity may negatively affect the contraction of actomyosin (19). Microtubules perform an essential role in maintaining cell polarity and extra-cellular transportation. The interaction between ROCK and Diaphanous-related formin (Dia) contribute to the regulation of cell polarity and canaliculus (20). The present study identified that ursolic acid significantly inhibited ROCK protein expression and elevated cleaved ROCK protein expression in LNCaP cells. Li et al (21) suggested that ursolic acid promotes the apoptosis of gastric cancer cells via the ROCK/PTEN pathway.

The importance of PTEN can be demonstrated by its frequent destruction to cancer cells (21). PTEN is the first known phosphatase which can inhibit tumor activity. In tumor cells, mechanisms which regulate the expression and functional changes of PTEN include the regulation of PTEN transcription, post-transcriptional regulation of encoding RNA, modification following interpretation and protein interactions (22). Slight changes to the expression levels of PTEN may influence the occurrence and progression of tumors (23). The present study demonstrated that ursolic acid significantly promoted p-PTEK protein expression in prostate cancer LNCaP cells. Wu et al (24) also reported that ursolic acid induced apoptosis in K562 cells by upregulating PTEN gene expression and cytochrome c. Li et al (21) suggested that ursolic acid promotes the apoptosis of gastric cancer cells through the ROCK/PTEN pathway.

Cofilin-1 is a fundamental regulatory factor in the invasion and metastasis of cancer cells (25). The overexpression of cofilin-1 increases the speed of tumor migration, and the inhibition of its expression can therefore significantly reduce the invasion of tumor cells $(6,9)$. The present study revealed that ursolic acid significantly enhanced cofilin-1 protein expression in cytoplasmic LNCaP cells. Li et al (21) reported that ursolic acid promotes the apoptosis of gastric cancer cells via ROCK/PTEN mediated cofilin-1 expression in the SGC-7901 cell line.

Cytochrome $\mathrm{c}$ is the control center for cell movement, and is not only the center of cellular respiratory chains and oxidative phosphorylation, but is also the regulatory center of cell apoptosis (26). The release of cytochrome c is a key step in cellular apoptosis. Under the conditions of deoxyadenosine triphosphate (dATP), cytochrome can combine with apoptotic protease activating factor 1 (APaf-1), which in turn promotes the formation of polymers and also enhances the formation of apoptosome (27). Activated caspase- 9 results in the activation of other caspases including caspase-3. Caspase-3 triggers cascade reactions of Caspases, which subsequently results in apoptosis (28). A new study demonstrated that ursolic acid significantly increased the protein expression of cytochrome 
c and augmented the activities of caspase- 3 and caspase- 9 in LNCaP cells (28). In addition, Shyu et al (12) suggested that ursolic acid can induce the apoptosis of human hepatocellular carcinoma cells via the activation of caspase-9 and caspase-3. Similarly, Park et al (16) suggested that ursolic acid induced apoptosis in prostate cancer PC-3 cells through caspase- 9 and caspase-3. Wu et al (24) also reported that ursolic acid induced apoptosis following the upregulation of PTEN and cytochrome c in K562 cells.

In conclusion, the present study demonstrates that ursolic acid activates the apoptosis of prostate cancer, at least in part by directly targeting ROCK/PTEN mediated mitochondrial translocation of cofilin-1. In future studies, the authors aim to focus on the drug development of ursolic acid for the treatment of human prostate cancer.

\section{References}

1. Hoare D, Skinner TA, Black A and Robert Siemens D: Serum follicle-stimulating hormone levels predict time to development of castration-resistant prostate cancer. Can Urol Assoc J 9: 122-127, 2015

2. Yoon BI, Shin TS, Cho HJ, Hong SH, Lee JY, Hwang TK and Kim SW: Is it effective to perform two more prostate biopsies according to prostate-specific antigen level and prostate volume in detecting prostate cancer? Prospective study of 10-core and 12-core prostate biopsy. Urol J 9: 491-497, 2012.

3. Ankerst DP, Till C, Boeck A, Goodman PJ, Tangen CM and Thompson IM: Predicting risk of prostate cancer in men receiving finasteride: Effect of prostate volume, number of biopsy cores and american urological association symptom score. Urology 82: 1076-1081, 2013

4. Yli-Hemminki TH, Laurila M, Auvinen A, Määttänen L, Huhtala H, Tammela TL and Kujala PM: Histological inflammation and risk of subsequent prostate cancer among men with initially elevated serum prostate-specific antigen (PSA) concentration in the Finnish prostate cancer screening trial. BJU Int 112: 735-741, 2013.

5. Zhu B, Fukada K, Zhu H and Kyprianou N: Prohibitin and cofilin are intracellular effectors of transforming growth factor beta signaling in human prostate cancer cells. Cancer Res 66: 8640-8647, 2006

6. Wang Y, Kuramitsu Y, Ueno T, Suzuki N, Yoshino S, Iizuka N, Zhang X, Oka M and Nakamura K: Differential expression of up-regulated cofilin-1 and down-regulated cofilin-2 characteristic of pancreatic cancer tissues. Oncol Rep 26: 1595-1599, 2011

7. Tang Q, Ji Q, Tang Y, Chen T, Pan G, Hu S, Bao Y, Peng W and Yin P: Mitochondrial translocation of cofilin-1 promotes apoptosis of gastric cancer BGC-823 cells induced by ursolic acid Tumour Biol 35: 2451-2459, 2014.

8. Li M, Yin J, Mao N and Pan L: Upregulation of phosphorylated cofilin 1 correlates with taxol resistance in human ovarian cancer in vitro and in vivo. Oncol Rep 29: 58-66, 2013.

9. Lu LI, Fu NI, Luo XU, Li XY and Li XP: Overexpression of cofilin 1 in prostate cancer and the corresponding clinical implications. Oncol Lett 9: 2757-2761, 2015.

10. Zang LL, Wu BN, Lin Y, Wang J, Fu L and Tang ZY: Research progress of ursolic acid's anti-tumor actions. Chin J Integr Med 20: 72-79, 2014.

11. Mazumder K, Tanaka K and Fukase K: Cytotoxic activity of ursolic acid derivatives obtained by isolation and oxidative derivatization. Molecules 18: 8929-8944, 2013.

12. Shyu MH, Kao TC and Yen GC: Oleanolic acid and ursolic acid induce apoptosis in $\mathrm{HuH} 7$ human hepatocellular carcinoma cells through a mitochondrial-dependent pathway and downregulation of XIAP. J Agric Food Chem 58: 6110-6118, 2010.
13. Wu HY, Chang CI, Lin BW, Yu FL, Lin PY, Hsu JL, Yen CH, Liao MH and Shih WL: Suppression of hepatitis B virus $\mathrm{X}$ protein-mediated tumorigenic effects by ursolic Acid. J Agric Food Chem 59: 1713-1722, 2011.

14. Ma JQ, Ding J, Xiao ZH and Liu CM: Ursolic acid ameliorates carbon tetrachloride-induced oxidative DNA damage and inflammation in mouse kidney by inhibiting the STAT3 and NF-кB activities. Int Immunopharmacol 21: 389-395, 2014.

15. Gontero P, Marra G, Soria F, Oderda M, Zitella A, Baratta F, Chiorino G, Gregnanin I, Daniele L, Cattel L, et al: A randomized double-blind placebo controlled phase I-II study on clinical and molecular effects of dietary supplements in men with precancerous prostatic lesions. Chemoprevention or 'chemopromotion'? Prostate 75: 1177-1186, 2015

16. Park JH, Kwon HY, Sohn EJ, Kim KA, Kim B, Jeong SJ, Song JH, Koo JS and Kim SH: Inhibition of Wnt/beta-catenin signaling mediates ursolic acid-induced apoptosis in PC-3 prostate cancer cells. Pharmacol Rep 65: 1366-1374, 2013.

17. Zhang J, Wang W, Qian L, Zhang Q, Lai D and Qi C: Ursolic acid inhibits the proliferation of human ovarian cancer stem-like cells through epithelial-mesenchymal transition. Oncol Rep 34: 2375-2384, 2015 .

18. Li Y, Li X, Liu KR, Zhang JN, Liu Y and Zhu Y: Visfatin derived from ascites promotes ovarian cancer cell migration through Rho/ROCK signaling-mediated actin polymerization. Eur J Cancer Prev 24: 231-239, 2015.

19. Sturge J, Wienke D and Isacke CM: Endosomes generate localized Rho-ROCK-MLC2-based contractile signals via Endo180 to promote adhesion disassembly. J Cell Biol 175: 337-347, 2006.

20. Richerioux N, Blondeau C, Wiedemann A, Rémy S, Vautherot JF and Denesvre C: Rho-ROCK and Rac-PAK signaling pathways have opposing effects on the cell-to-cell spread of Marek's disease virus. PLoS One 7: e44072, 2012

21. Li R, Wang X, Zhang XH, Chen HH and Liu YD: Ursolic acid promotes apoptosis of SGC-7901 gastric cancer cells through ROCK/PTEN mediated mitochondrial translocation of cofilin-1. Asian Pac J Cancer Prev 15: 9593-9597, 2014.

22. Pabona JM, Dave B, Su Y, Montales MT, de Lumen BO, de Mejia EG, Rahal OM and Simmen RC: The soybean peptide lunasin promotes apoptosis of mammary epithelial cells via induction of tumor suppressor PTEN: Similarities and distinct actions from soy isoflavone genistein. Genes Nutr 8: 79-90, 2013.

23. Sfakianos JP, Lin Gellert L, Maschino A, Gotto GT, Kim PH, Al-Ahmadie $\mathrm{H}$ and Bochner BH: The role of PTEN tumor suppressor pathway staining in carcinoma in situ of the bladder. Urol Oncol 32: 657-662, 2014.

24. Wu B, Wang X, Chi ZF, Hu R, Zhang R, Yang W and Liu ZG: Ursolic acid-induced apoptosis in K562 cells involving upregulation of PTEN gene expression and inactivation of the PI3K/Akt pathway. Arch Pharm Res 35: 543-548, 2012.

25. Atefi M, Avramis E, Lassen A, Wong DJ, Robert L, Foulad D, Cerniglia M, Titz B, Chodon T, Graeber T, et al: Effects of MAPK and PI3K pathways on PD-L1 expression in melanoma. Clin Cancer Res 20: 3446-3457, 2014.

26. Banafa AM, Roshan S, Liu YY, Chen HJ, Chen MJ, Yang GX and He GY: Fucoidan induces G1 phase arrest and apoptosis through caspases-dependent pathway and ROS induction in human breast cancer MCF-7 cells. J Huazhong Univ Sci Technolog Med Sci 33: 717-724, 2013.

27. Balachandran C, Sangeetha B, Duraipandiyan V, Raj MK, Ignacimuthu S, Al-Dhabi NA, Balakrishna K, Parthasarathy K, Arulmozhi NM and Arasu MV: A flavonoid isolated from Streptomyces sp. (ERINLG-4) induces apoptosis in human lung cancer A549 cells through p53 and cytochrome c release caspase dependant pathway. Chem Biol Interact 224: 24-35, 2014.

28. Li HH, Su JH, Chiu CC, Lin JJ, Yang ZY, Hwang WI, Chen YK, Lo $\mathrm{YH}$ and $\mathrm{Wu} \mathrm{YJ}$ : Proteomic investigation of the sinulariolide-treated melanoma cells A375: Effects on the cell apoptosis through mitochondrial-related pathway and activation of caspase cascade. Mar Drugs 11: 2625-2642, 2013. 